\title{
Finite State Machine State Assignment for Area and Power Minimization
}

\author{
Aiman El-Maleh, Sadiq M. Sait and Faisal Nawaz Khan \\ Department of Computer Engineering \\ King Fahd University of Petroleum \& Minerals, Saudi Arabia \\ Email: \{aimane, sadiq, fnawaz\} @ ccse.kfupm.edu.sa
}

\begin{abstract}
In this paper, we address the problem of FSM state assignment to minimize area and power. The objectives are targeted as single/independent as well as multi-objective optimization (MOP) problems. Novel methods for estimating area and power of an FSM are presented. A fuzzy-based aggregation function is employed to combine the two objectives. The work employs genetic algorithm for search space exploration. Experimental results demonstrate the effectiveness of the proposed measures. ${ }^{1}$
\end{abstract}

\section{INTRODUCTION}

State assignment (SA) for Finite State Machine (FSM) is one of the main optimization problems in the synthesis of sequential circuits. The SA of an FSM determines the complexity of its combinational circuit and thus area and power dissipation of the implementation. State assignment involves an injective mapping $\mathrm{f}: \mathrm{S} \rightarrow B^{n}$ where $n$ is the code length $\left(n \geq\left\lceil\log _{2}|S|\right\rceil\right)$ and $B^{n}$ is an $n$-dimensional Boolean space, a Boolean hypercube.

The objective of state assignment varies depending on whether the targeted implementation is two-level or multilevel. The focus of this work is towards minimizing multilevel FSM implementation.

The complexity measure for multilevel circuits is the number of literals in the optimized logic network assuming minimum encoding length. Literal savings by extracting common subexpressions has been the focus of most of the work done for multilevel FSM optimization. This involves finding pairs of states that when encoded can result in extraction of common subexpressions. In contrast to two-level circuits, state pairs in multilevel implementations do not necessarily have to be given adjacent codes for literal savings [1]-[3].

The problem of multilevel area minimization of an FSM has been modeled as weighted [1]-[3] or constrained [4]-[6] graph-embedding problem on Boolean hypercubes.

Portable electronics applications have given power-aware computing a whole new importance. This is due to limitations in battery capacities and to the fact that progress in their technologies trail far behind the ever increasing computing requirements. Power consumption is thus constrained and optimized at all levels of design hierarchy [7]. The major source of power consumption in CMOS circuits is due to charging and discharging of circuit capacitances. Power consumption in a circuit can be reduced by either reducing the total switching

\footnotetext{
${ }^{1}$ This work is supported by King Fahd University of Petroleum \& Minerals.
}

in the logic or by reducing the switching capacitance or both of them.

There has been much interest in power reduction strategies for FSM. Most of the work reported in literature [8]-[14] tries to minimize total switching on the flip-flops. This is done by formulating the problem as a graph embedding problem where edges between a state pair indicate the steady state transition probability between them. The problem is thus reduced to minimizing the total transitions between the states. Such a formulation is referred to as Minimum Weighted Hamming Distance (MWHD).

The hard nature of SA problem has generated considerable interest in the use of non-deterministic heuristics such as genetic algorithm [3], [12] and simulated annealing [1], for its design automation. These algorithms are capable of efficiently searching for a near optimal solution in a large solution space and have been very successful in solving a number of combinatorial optimization problems in various disciplines of science and engineering.

In this paper, we explore the use of genetic algorithm for SA problem where the logic synthesized is implemented as multilevel circuit. We present novel and efficient strategies for estimating multilevel area and power dissipation in an FSM. We also evaluate several integration mechanisms for combining area and power measures.

\section{Proposed Methodology}

\section{A. State Assignment for Area}

The contemporary approach employed towards multilevel FSM area minimization is by using weighted-graph approach where weights between edges of states define the relative proximity in assignment (affinity).

Affinity cost as modeled in adjacency graphs is then used to minimize Equation 1.

$$
\sum_{i=1}^{n_{s}} \sum_{j=1}^{n_{s}} A_{S_{i}, S_{j}}^{P} \cdot \Delta(i, j)
$$

where $\Delta\left(S_{i}, S_{j}\right)$ is the Hamming distance between codes of state $i$ and $j, A_{i, j}^{P}$ being the affinity between two states as given by the used cost measure.

In [15], several literal saving measures based on weightedgraph embedding problem including those based on Jedi [1], Mustang [2] and Armstrong [16] cost measures were 
investigated. It was found that all these measures weakly correlate with the actual literal savings measure.

There are several complications in using weighted graph approach for literal savings measure estimation based on common cube extraction. First, the common cubes interact with each other, annulling certain predicted savings. Second, such measures optimize relative literal savings rather than absolute number of literals in an implementation. Consequently, they may lead the search to solutions having higher relative savings with higher implementation costs. Such an interaction is very difficult to predict at higher level of abstractions.

In order to use an efficient but accurate estimate of actual literal savings, we propose the use of EXPAND function, employed in ESPRESSO [17], as a measure for estimating area of an FSM. EXPAND is the first step employed in two-level logic minimization and provides a cover for the function that is minimal with respect to single-cube containment. However, the cover might contain some redundant terms. It should be observed that ESPRESSO [17] is based on iterative application of several functions including EXPAND, IRREDUNDANT and REDUCE. Hence, using EXPAND as a cost measure is more efficient than using ESPRESSO. As will be illustrated by experimental results in Section III, it is found that using EXPAND with single output optimization strongly correlates with actual multilevel literal savings measure.

\section{B. State Assignment for Low Power}

Switching activity in combinational logic of the controllers is due to logic transitions on the flip-flops as well as on primary inputs. Thus, to reduce power dissipation in an FSM, one can:

1) Minimize switching activity at the flip-flops.

2) Minimize the capacitance on flip-flops being switched, i.e., fanout branches (fanout) from flip-flops.

3) Minimize the combinational logic being switched.

State assignment for power minimization can also be modeled by a weighted graph where weights between edges of states represent the total switching probability between the two states. By assigning shorter distance codes to states connected with higher weights, i.e., higher transition probability, the overall switching on the state lines of the FSM can be minimized. Thus, a cost model for minimizing power consumption can be to have Minimum Weighted Hamming Distance (MWHD). Mathematically, this can be achieved by minimizing Equation 1 with the affinity being modeled as:

$$
A_{S_{i}, S_{j}}^{P}=P_{i j}+P_{j i}
$$

where $P_{i j}$ is the state transition probability from state $S_{i}$ to state $S_{j}$.

The first formulation for power minimization used in this work tries to optimize the above MWHD cost. MWHD approach tries to minimize the total transition probability of the state machine in the hope that the total number of logic transitions in the synthesized circuit will also get reduced, i.e., it tries to maximize power reductions only due to point 1 discussed above.

In this work, we propose a new cost function for minimizing power dissipation in an FSM. The idea is based on minimizing fanout branches out of frequently switched sequential elements. Reducing fanout branches on highly switching flipflops reduces the switching capacitance and hences reduces power dissipation. The frequency of sequential elements' transitions are computed based on steady state transition probabilities. A logic transition in the assignments for a sequential element between two connected states represent transition activity on that sequential element. The flip-flops' transition frequencies are weighted with their respective fanout counts for determining minimum weighted fanout solution by minimizing Equation 3:

$$
\text { Fanout }=\sum_{i=1}^{n_{E}} T_{i} B_{i}
$$

where $B_{i}$ and $T_{i}$ are the number of fanout branches and the transition frequencies of flip-flop $i$, respectively. Expand cover is used in estimating flip-flop fanout branches.

\section{Genetic Algorithm}

Genetic algorithms have been adopted to explore the solution space in search of good state assignments. The chromosomal representations employed and the crossover operator used is similar to the one suggested by Amaral et al. [3]. In this representation, each state code is described as an array of bits equal to the number of storage elements required. Parent selection for crossover is based on the roulette wheel method. Crossover is performed by randomly selecting a subset of state encoding columns from the first parent and the rest from the second parent. The mutation operator used swaps two randomly selected states in a randomly selected parent. The number of mutations occurring in a generation is controlled by the mutation rate. A value of $20 \%$ is selected and mutation is applied to all but the best solution.

After every generation, members for the next generation are selected from both parents and newly generated offsprings. A combination of greedy (for the best half of parents and new offsprings) and random from the second half is used. Diversity in population is maintained by discarding duplicate offsprings. In this work, a population size of 64 and a maximum generation size of 350 produced best results.

The area and power objectives are aggregated using product based and Ordered Weighted Averaging (OWA) operator [18]. In OWA, we employ both "orlike" (Max) and "andlike" (Min) fuzzy operators as given in Equation 4.

$$
\mu=\beta \times O\left(\mu_{a}, \mu_{p}\right)+(1-\beta) \times \frac{1}{2}\left(\mu_{a}+\mu_{p}\right)
$$

where $O$ is $\max / \mathrm{min}$ type fuzzy operator, $\mu_{i}$ represents cost for area or power objective and $\beta$ is a constant parameter that represents the degree to which OWA operator resembles a pure "or" or pure "and" respectively. In this work we employ $\beta=0.5$ 
TABLE I

COMPARISON OF EXPAND-SO MEASURE WITH OTHER AREA MINIMIZATION HEURISTICS.

\begin{tabular}{|c|c|c|c|c|c|c|c|}
\hline Benchmark & EXPAND-SO & ESPRESSO-SO+FX & EXPAND-MO & Jedi ( [1] ) & Nova & Mustang [2] & Armstrong( [3] ) \\
\hline bbara & $56(52)$ & 51 & 57 & $73(57)$ & 57 & 64 & $59(86)$ \\
bbsse & $110(105)$ & 103 & 120 & $134(111)$ & 140 & 106 & $127(180)$ \\
cse & $198(228)$ & 183 & 239 & $240(200)$ & 214 & 206 & $220(\mathrm{NA})$ \\
dk14 & $104(86)$ & 98 & 115 & $108(76)$ & 111 & 117 & $124(\mathrm{NA})$ \\
donfile & $87(72)$ & 49 & 106 & $82(76)$ & 154 & 160 & $171(\mathrm{NA})$ \\
ex2 & $78(68)$ & 66 & 130 & $123(122)$ & 127 & 119 & $131(\mathrm{NA})$ \\
ex3 & $56(48)$ & 53 & 67 & $65(66)$ & 71 & 71 & $68(\mathrm{NA})$ \\
keyb & $199(205)$ & 134 & 161 & $260(140)$ & 201 & 167 & $334(\mathrm{NA})$ \\
lion9 & $11(11)$ & 10 & 25 & $19(13)$ & 27 & 17 & $27(21)$ \\
planet & $486(436)$ & 439 & 557 & $603(547)$ & 591 & 544 & $607(\mathrm{NA})$ \\
pma & $165(152)$ & 153 & 189 & $263(\mathrm{NA})$ & 241 & NA & $218(\mathrm{NA})$ \\
s1 & $227(105)$ & 155 & 285 & $282(152)$ & 340 & 183 & $291(\mathrm{NA})$ \\
s1494 & $570(624)$ & 543 & 717 & $679(\mathrm{NA})$ & 715 & NA & $696(\mathrm{NA})$ \\
s832 & $231(218)$ & 215 & 307 & $357(\mathrm{NA})$ & 274 & NA & $301(\mathrm{NA})$ \\
sand & $498(494)$ & 473 & 514 & $554(437)$ & 558 & 462 & $619(\mathrm{NA})$ \\
shiftreg & $2(2)$ & 2 & 4 & $2(2)$ & 2 & 2 & $2(10)$ \\
styr & $419(429)$ & 379 & 466 & $518(508)$ & 502 & 546 & $546(\mathrm{NA})$ \\
tbk & $353(268)$ & 312 & 493 & $305(278)$ & 365 & 547 & $711(\mathrm{NA})$ \\
traian11 & $22(20)$ & 18 & 29 & $34(27)$ & 32 & 37 & $32(47)$ \\
\hline \%Improvement & & -12.69 & 15.48 & $17.63(6.51)$ & 18 & 22.56 & $26.72(41.57)$ \\
\hline
\end{tabular}

\section{EXPERIMENTAL RESULTS}

In this section, experimental results of the proposed state assignment measures for area and power minimization are presented. Experiments are performed on a subset of MCNC93 FSM benchmark circuits with different complexities.

\section{A. Area}

In Table I, we compare the performance of several cost functions targeting area minimization including Espresso with single-output minimization followed by FX (ESPRESSO$\mathrm{SO}+\mathrm{FX}$ ), Expand with single-output minimization (EXPANDSO), Expand with multiple-output minimization (EXPANDMO), Jedi [1], Nova [6], Mustang [2], and Armstrong [3].

The values in the table represent the literal count obtained after synthesizing the obtained solutions using ESPRESSO$\mathrm{SO}+\mathrm{FX}$. Results shown in brackets are those reported in literature based on synthesis using script.rugged except for Amaral et al. [3] where results are reported in factored form.

The last row in the table shows the percentage improvement of EXPAND-SO over all other cost measures. It is observed that EXPAND-SO achieves significant improvements over all other techniques and only lags behind the accurate cost measure using ESPRESSO-SO+FX by nearly $13 \%$.

\section{B. Power}

The performance of the proposed Fanout measure is next compared with MWHD and default Jedi state assignment algorithm in Table II. Power consumptions are measured in microwatts using power_estimate -t SEQUENTIAL option of SIS and assuming default conditions. It can be seen that the Fanout measure achieves better results than MWHD and Jedi on both area and power, and on average it achieves $6.47 \%$ less power and $14.53 \%$ less area, per circuit, than Jedi algorithm.

The multiobjective optimization of both area and power is shown by integrating the Expand-SO area estimate with Fanout measure. We employ both the product of area and power estimates $(F A)$ as well as fuzzy-based aggregations, employing Max and Min type fuzzy operators using equal weighted OWA aggregation. As can be seen from the results, the multiobjective optimization of both area and power achieves overall higher reduction in both area and power with Fanout(FuzzyMax) resulting in the least power dissipation.

\section{Literature Comparison}

In Table III, we compare the performance of the Fanout(Fuzzy-Max) measure to other techniques reported in literature [10], [12]-[14]. Comparison is made relative to the output-oriented (default) Jedi state assignment algorithm, with results reported as \%reduction relative to Jedi. The last row in the table shows the percentage improvement achieved by our technique over other techniques.

It can be seen that our approach achieves higher overall savings over all the approaches. The Fanout(Fuzzy-Max), though being slightly better in power dissipation, significantly outperforms Pedram's approach [10] in area savings. Significant power savings are also observed between our approach and Ciesielski et al. [13] and IITG8 [14] measures. The ingenuity of our measure can also be seen from its complete dominance over Almiani et al.'s approach [12] in both area and power optimizations, although the latter employs a more expensive Espresso iteration in their cost calculation.

\section{CONCLUSION}

In this work, we have presented a genetically engineered state assignment solution for area and power minimization. We have proposed efficient cost functions that highly correlate with the actual literal count and power dissipation of a multilevel circuit implementation. Experimental results demonstrate the effectiveness of the proposed measures in achieving lower area and power dissipation solutions in comparison to techniques reported in the literature.

\section{REFERENCES}

[1] B. Lin and A. R. Newton. Synthesis of multiple-level logic from symbolic high-level description languages. IFIP International Conference on Very Large Scale Integration, pages 187-196, August 1989. 
TABLE II

AREA AND POWER DISSIPATION FOR VARIOUS HEURISTICS.

\begin{tabular}{|c|c|c|c|c|c|c|c|c|c|c|c|c|}
\hline \multirow[b]{2}{*}{ Benchmark } & \multicolumn{2}{|c|}{ MWHD } & \multicolumn{2}{|c|}{ Fanout } & \multicolumn{2}{|c|}{ Jedi } & \multicolumn{2}{|c|}{ FA } & \multicolumn{2}{|c|}{ Fanout(Fuzzy-Max) } & \multicolumn{2}{|c|}{ Fanout(Fuzzy-Min) } \\
\hline & Power & Area & Power & Area & Power & Area & Power & Area & Power & Area & Power & Area \\
\hline bbara & 214.7 & 82 & 150.5 & 55 & 187.7 & 74 & 181.2 & 65 & 181.2 & 58 & 181.2 & 58 \\
\hline bbsse & 446.1 & 140 & 412.2 & 122 & 538.8 & 149 & 394.5 & 118 & 437.1 & 123 & 448.2 & 128 \\
\hline cse & 528.9 & 217 & 424.8 & 211 & 495.8 & 251 & 391.3 & 209 & 455.2 & 205 & 459.9 & 210 \\
\hline dk14 & 661.2 & 140 & 561.4 & 103 & 714.4 & 157 & 561.4 & 103 & 551.3 & 101 & 579.2 & 115 \\
\hline donfile & 895.9 & 206 & 513.7 & 109 & 380.8 & 89 & 474.1 & 100 & 355.2 & 82 & 295.8 & 75 \\
\hline keyb & 655.3 & 263 & 645 & 237 & 767.6 & 260 & 517.3 & 215 & 535.6 & 210 & 511.7 & 192 \\
\hline lion9 & 142 & 20 & 116.7 & 19 & 145.6 & 19 & 100.8 & 15 & 105.3 & 16 & 129.9 & 11 \\
\hline planet & 1788.6 & 656 & 1795.1 & 553 & 2001.5 & 675 & 1889.7 & 510 & 1702.5 & 470 & 1843.2 & 465 \\
\hline pma & 653.4 & 198 & 778 & 180 & 883.7 & 236 & 693.1 & 165 & 651.2 & 155 & 675.2 & 145 \\
\hline s1 & 1165.1 & 406 & 766.5 & 187 & 1205.3 & 353 & 771.4 & 197 & 751.2 & 191 & 625.8 & 161 \\
\hline s1494 & 1376.3 & 734 & 1553.1 & 625 & 1668.9 & 679 & 852.4 & 569 & 838.2 & 530 & 1025.5 & 505 \\
\hline s832 & 922.1 & 368 & 677.5 & 271 & 1068.4 & 376 & 665.2 & 260 & 650.2 & 242 & 621.5 & 249 \\
\hline sand & 1645.5 & 599 & 1541.4 & 559 & 1458.9 & 651 & 1617.2 & 585 & 1289.9 & 490 & 1352.2 & 498 \\
\hline shiftreg & 163.3 & 27 & 96.3 & 2 & 132.5 & 9 & 98.8 & 4 & 96.3 & 2 & 96.3 & 2 \\
\hline styr & 1277.5 & 540 & 1062.9 & 431 & 1118.6 & 567 & 1086.8 & 453 & 1022.3 & 432 & 1100 & 376 \\
\hline tbk & 1682 & 630 & 1589.3 & 488 & 721.2 & 305 & 1766.6 & 556 & 1095.2 & 422 & 1350 & 398 \\
\hline train11 & 180.4 & 38 & 136.3 & 23 & 218.2 & 35 & 142.4 & 22 & 122.2 & 23 & 150.1 & 21 \\
\hline Average & 846.96 & 309.65 & 754.16 & 245.59 & 806.35 & 287.35 & 717.89 & 243.88 & 637.65 & 220.71 & 673.28 & 212.29 \\
\hline
\end{tabular}

TABLE III

POWER AND AREA \%-REDUCTION COMPARISON WITH JEDI.

\begin{tabular}{|c|c|c|c|c|c|c|c|c|}
\hline & \multicolumn{2}{|c|}{ Fanout(Fuzzy-Max) } & \multicolumn{2}{|c|}{ Pedram } & \multirow{2}{*}{$\begin{array}{c}\text { Ciesielski [13] } \\
\text { Power }\end{array}$} & \multirow{2}{*}{$\begin{array}{c}\text { IITG8 [14] } \\
\text { Power }\end{array}$} & \multicolumn{2}{|c|}{ Almaini } \\
\hline & Power & Area & Power & Area & & & Power & Area \\
\hline bbara & 3.46 & 21.62 & 17.97 & -10.14 & & 16.07 & -25.68 & 21.62 \\
\hline bbsse & 18.88 & 17.45 & 18.37 & 6.56 & 5.66 & & & \\
\hline cse & 8.19 & 18.33 & 12.15 & -1.41 & & 18.48 & 2.58 & 18.33 \\
\hline dk14 & 22.83 & 35.67 & 4.92 & -0.98 & & 16.19 & & \\
\hline donfile & 6.72 & 7.86 & 6.22 & 22.64 & & & -5.57 & 7.86 \\
\hline keyb & 30.22 & 19.23 & & & 35.56 & 20.87 & 2.53 & 19.23 \\
\hline lion9 & 27.68 & 15.79 & & & & & & \\
\hline planet & 14.93 & 30.37 & & & & & -19.22 & 30.37 \\
\hline pma & 26.3 & 34.32 & & & & & & \\
\hline s1 & 37.68 & 45.89 & & & & -22.46 & -7.32 & 45.89 \\
\hline s1494 & 49.78 & 21.94 & & & 6.89 & & & \\
\hline s832 & 39.14 & 35.64 & & & 7.75 & 26.68 & & \\
\hline sand & 11.58 & 24.73 & 10.52 & 16.12 & & & -19.29 & -30.32 \\
\hline shiftreg & 27.32 & 77.78 & & & & -29.08 & & \\
\hline styr & 8.61 & 23.81 & & & & -9 & 9.16 & 23.81 \\
\hline tbk & -51.86 & -38.36 & & & 5.03 & & & \\
\hline train 11 & 44 & 34.28 & & & & 11.61 & 13.2 & 34.29 \\
\hline \%Improvement & & & 2.09 & 73.9 & 29.31 & 74.74 & 130 & 24.36 \\
\hline
\end{tabular}

[2] S. Devadas, H.T. Ma, A.R. Newton, and Sangiovanni-Vincentelli. MUSTANG: State Assignment of Finite State Machines for Optimal MultiLevel Logic Implememations. IEEE Transactions on Computer-Aided Design of Integrated Circuits and Systems, 7:1290-1300, Dec. 1988.

[3] J. Amaral, K. Turner, and J. Ghosh. Designing Genetic Algorithm for State Assignment Problem. IEEE Trans on SMC, 25:659-694, 1995.

[4] G. DeMicheli, R. K. Brayton, and A. Sangiovanni Vincenteli. Optimal state assignment for Finite State Machines. IEEE Transaction on Computer Aided Design, CAD-4:3, 269-285, 1985.

[5] X. Du, G. Hachtel, B. Lin, and A. R. Newton. MUSE: A Multilevel symbolic encoding algorithm for state assignment. IEEE Trans. Computer Aided Design, CAD-10:28-38, 1991.

[6] T. Villa and A. Sangiovanni-Vincentelli. Nova: state assignment of finite state machines for optimal two-level logic implementations. Proceedings of the 1989 26th ACM/IEEE conference on Design automation conference, pages 327-332, 1989.

[7] M. Pedram. Design technologies for low power VLSI. In Encyclopedia of Computer Science and Technology, Marcel Dekker, Inc., pages 73-96, 1997.

[8] L. Benini and G. DeMicheli. State encoding for low power embedded controllers. IEEE Journal of Solid-State Circuits, 30:258-268, 1995.

[9] E. Olson and S. M. Kang. State assignment for low-power FSM synthesis using genetic local search. IEEE Custom Integrated Circuits Conference, pages 140-143, 1994.

[10] Chi-Ymg Tsui, M. Pedram, Chih-Ang Chen, and A. M. Despain. Low-Power State Assignment Targeting Two And Multi-level Logic Implementations. IEEE/ACM International Conference on ComputerAided Design, pages 82-87, 1994.
[11] V. Vamshi, T. Akhilesh, and R. Suresh. Re-encoding for Low Power State Assignment of FSMs. ACM International Symposium on Low Power Design, pages 173-178, 1995.

[12] Y. Xia and A. E. A. Almaini. Genetic algorithm based state assignment for power and area optimisation. IEEE Proceedings Computers and Digital Techniques, 149:128-133, 2002.

[13] Sangju. Park, Sangwook Cho, Seiyang Yang, and Maciej Ciesielski. A New State Assignment Technique for Testing and Low Power. Annual ACM IEEE Design Automation Conference, pages 510-513, 2004.

[14] S. Chattopadhyay and P. N. Reddy. Finite state machine state assignment targeting low power consumption. IEEE Proceedings Computers and Digital Techniques, 151:61-70, 2004.

[15] Faisal N. Khan. FSM State Assignmnet for Area, Power and Testability using Non-Deterministic Evolutionary Heuristics. Master's thesis, KFUPM, Saudi Arabia, 2005.

[16] D. B Armstrong. A programmed algorithm for assigning internal codes to sequential machines. IRE Transactions on Electronic Computers, pages 466-472, 1962.

[17] Robert K. Brayton, Gary D. Hachtel, Curtis T. McMullen, and Alberto L. Sangiovanni-Vincentelli. Logic Minimization Algorithms for VLSI Synthesis. Kluwer Academic Publishers, 1984.

[18] R. Yager. Multiple Objective Decision-making Using Fuzzy Sets. International Journal of Man-Machine Studies, pages 9:375-382, 1977. 